\title{
Novel Mumps Virus Epitopes Reveal Robust Cytotoxic T Cell Responses After Natural Infection but Not After Vaccination
}

Patricia Kaaijk ( $\nabla$ patricia.kaaijk@rivm.nl )

National Institute for Public Health and the Environment

Maarten Emmelot

National Institute for Public Health and the Environment

Hugo Meiring

Intravacc (Netherlands)

Cecile van Els

National Institute for Public Health and the Environment

Jelle de Wit

National Institute for Public Health and the Environment

\section{Research Article}

Keywords: Mumps virus, T cell epitopes, T cell response, cytotoxic CD8+ T-cells, cellular immunity, MHC class I, mumps virus infection, measles-mumps-rubella vaccination

Posted Date: January 22nd, 2021

DOI: https://doi.org/10.21203/rs.3.rs-147373/v1

License: (9) (1) This work is licensed under a Creative Commons Attribution 4.0 International License. Read Full License 


\section{Abstract}

Mumps is nowadays re-emerging despite vaccination. The contribution of T cell immunity to protection against mumps has not been clearly defined. Previously, we described a set of 41 peptides that were eluted from human leukocyte antigen (HLA) class I molecules of mumps virus (MuV)-infected cells. Here, we confirmed immunogenicity of five novel HLA-B*07:02- and HLA-A*01:01-restricted MuV T cell epitopes from this set of peptides. High frequencies of T cells against these five MuV epitopes could be detected ex vivo in all tested mumps patients. Moreover, these epitope-specific T cells derived from mumps patients displayed strong cytotoxic activity. In contrast, only marginal T cell responses against these novel MuV epitopes could be detected in recently vaccinated persons, corroborating earlier findings. Identifying which MuV epitopes are dominantly targeted in the mumps-specific CD8 ${ }^{+} \mathrm{T}$ - response is an important step towards better understanding in the discrepancies between natural infection or vaccination-induced cell-mediated immune protection

\section{Introduction}

The implementation of routine measles-mumps-rubella (MMR) vaccination has led to a dramatic reduction in incidence of these infectious diseases, however, mumps is re-emerging nowadays ${ }^{1,2}$. Especially young adults that were vaccinated during childhood can be affected by mumps. Waning of vaccine-induced immunity in the absence of natural boosters by circulating mumps virus (MuV) has been suggested as major cause ${ }^{3-6}$. Various studies have shown a decline of antibody levels in time following childhood vaccination $5,7,8$. However, the finding that antibodies alone do not fully correlate with protection against MuV infection indicates that, apart from the humoral response, other immune compartments play a role in the immunity against $\mathrm{MuV}^{6,9,10}$. $\mathrm{T}$ cell responses against MuV have been detected in mumps cases as well as in vaccinated healthy persons ${ }^{9-12}$. Previously, we showed that strong MuV-specific CD8 ${ }^{+} \mathrm{T}$ cells dominate the T cell response in mumps patients. In the same study, we showed that the $\mathrm{CD} 8^{+} \mathrm{T}$ cell response in children that were recently vaccinated with the MMR vaccine was considerably less vigorous than after natural infection ${ }^{9}$. Several scientists have emphasized the importance of fully understanding the $\mathrm{T}$ cell immune responses to $\mathrm{MuV}$ after vaccination, which is needed to be able to develop strategies to improve the quality and durability of vaccine-induced immunity 2,13-15. For the detailed characterization of the $\mathrm{T}$ cell immune response to MuV and the understanding of its role in natural infection or vaccination, the identification of MuV-specific T cell epitopes is indispensable. Furthermore, to be able to investigate the cross-reactive potency of the $T$ cells induced by vaccination towards the circulating strains, data on the T cell response at the epitope level is also crucial.

Recently, a set of 41 unique viral peptides was eluted from HLA class I molecules of MuV-infected cells (typing: HLA-A*01:01, $A * 02: 01, B * 07: 02, B \star 40: 01, C * 03: 04, C * 07: 02$ ) and characterized by mass spectrometry. From this panel, we confirmed the first six HLA-A*02:01-restricted MuV T cell epitopes to be able to induce a T-cell response ${ }^{16}$. To expand our knowledge on the $\mathrm{CD} 8^{+} \mathrm{T}$ cell repertoire of MuV, we here searched for novel $\mathrm{T}$ cell epitopes among the earlier identified panel of naturally processed peptides 
with high predicted binding scores to the two other common HLA-I molecules, HLA-B*07:02 and HLA$A * 01: 01$. We describe five novel HLA-B*07:02- and HLA-A*01:01-restricted MuV epitopes that reveal for the first time robust ongoing cytotoxic $T$ cell responses in all mumps patients tested, with only low frequencies of epitope-specific T cells in recently vaccinated persons.

\section{Results}

In the present study, peptides from a previously characterized set of 41 naturally processed MuV epitopes 16 , predicted to bind best to HLA-B*07:02 $(n=12)$ and HLA-A*01:01 molecules $(n=10)$ were selected for immunogenicity assessment. Selected epitopes were tested for their capacity to recall a specific $T$ cell response, by using expanded $C D 8+$ T-effector cells isolated from three HLA-B*07:02 positive and three HLA-A*01:01 positive mumps patients (Fig. 1).

Three T cell epitopes, i.e., APIQGTNLL (phosphoprotein, aminoacid positions $27-35$ ) $\mathrm{V} / \mathrm{P} / \mathrm{I}_{27-35}$; KPRTSTPVTEF, V/P/I $142-152$; IPNARANL, (nucleoprotein) $\mathrm{NP}_{115-122}$, were identified that all elicit a $\mathrm{T}$ cell response in 3 out of 3 HLA-B*07:02 positive mumps patients, as measured by IFN-y and TNF production. In addition, three HLA-A*01:01-restricted epitopes, i.e., YSDPNNHEVY, (large protein) $\mathrm{L}_{1336-1343}$; YSDPNDHEVY, $L_{1336-1343}$; VTDSNLIY, $L_{1983-1992}$, induced a T cell response. The YSDPNNHEVY peptide, and its deamination variant, YSDPNDHEVY (in which the aspargine $(\mathrm{N})$ on position 6 was deaminated to aspartic acid (D)), both induced a T cell response in 3 out of 3 HLA-A $A^{\star} 0101$ positive mumps patients. The other HLA-A*01:01-restricted epitope (VTDSNLIY) induced IFN- $y$ and TNF production by $\mathrm{CD}^{+}{ }^{+}$effector T cells from 2 out 3 HLA-A*01:01 positive mumps patients (Fig. 1). Subsequently, APIQGTNLL, KPRTSTPVTEF, IPNARANL (HLA-B*07:02 restricted epitopes), and YSDPNNHEVY and VTDSNLIY (HLA$A \star 01: 01$ restricted epitopes) were selected for custom-made (p:HLA) dextramer synthesis to investigate the ex vivo occurrence of $\mathrm{CD}^{+} \mathrm{T}$ cells against these epitopes in mumps patients. YSDPNNHEVY and not its post-translationally modified deamination variant, YSDPNDHEVY, was selected because it was more abundantly presented based on elution from MHC-I molecules from MuV-infected cells ${ }^{16}$ and had a better predictive binding to HLA-A*01:01 (Fig. 1).

Using p:HLA dextramers, high frequencies of MuV epitope-specific T cells were observed in all three $B^{\star} 07: 02$ - and three HLA-*0101-positive patients, even exceeding $2 \%$ of the total CD ${ }^{+} T$ cell pool (Fig. $2 A$ ). The frequencies of the epitope-specific $\mathrm{CD} 8^{+} \mathrm{T}$ cells and the dominance of the response per epitope varied between patients. Further analyses showed that the majority peptide-specific $T$ cells were within the T-effector cell subset $\left(\mathrm{CD}^{+}{ }^{+} \mathrm{CD} 8^{+} \mathrm{CD} 45 \mathrm{RO}^{-} \mathrm{CCR} 7^{-}\right)$. Functional characterization revealed that, upon stimulation with peptides, the epitope-specific T cells expressed the activation marker CD137 and displayed a cytotoxic phenotype by expression of CD107a. In addition to pro-inflammatory cytokines IFN$Y$, TNF and IL-17A, epitope-specific T cells produced granzyme-A/B, granulysin, and to a lesser extent sFas $(L)$, and perforin that are involved in cell-mediated cytotoxicity (Fig. 2B). Finally, we showed that epitope-specific $T$ cells were able to efficiently lyse peptide-pulsed antigen-presenting target cells in a flow cytometry-based cytotoxicity assay (Fig. 2C). 
In contrast to the high frequencies of MuV epitope-specific T cells observed in mumps patients, only very low frequencies of MuV epitope-specific T cells could be detected in young adults that were recently vaccinated with a third dose of the MMR vaccine (Fig. 3). Despite the fact that epitope-specificity varied between individuals, in all tested PBMC samples of HLA-B7- and HLA-A1-positive vaccinated persons, small amounts of MuV epitope-specific CD8 ${ }^{+} \mathrm{T}$ cells could be visualized with the $\mathrm{p}$ :HLA dextramers.

\section{Discussion}

Identification of more functional $\mathrm{CD} 8^{+} \mathrm{T}$ cell epitopes that cover a broad repertoire of $\mathrm{T}$ cells against mumps virus $(\mathrm{MuV})$ is important for monitoring and functionally characterizing the $\mathrm{T}$ cell response following mumps infection or vaccination. Based on HLA-I binding prediction, most of the set of $41 \mathrm{MuV}$ peptides, from an earlier identified panel ${ }^{16}$, bound well to the HLA-B*07:02 molecule, followed by the HLA-A*02:01 and HLA-A ${ }^{\star}$ 01:01 molecules, i.e. belonging to the most common HLA-I alleles ${ }^{17}$. In addition to the previously identified HLA-A*02:01 epitopes, we now confirmed three HLA-B*07:02-restricted T cell epitopes (i.e. APIQGTNLL (V/P/I $27-35)$, KPRTSTPVTEF (V/P/I $142-152)$, IPNARANL $\left(\mathrm{NP}_{115-122}\right)$ ), each inducing a strong $T$ cell response in expanded effector $T$ cells obtained from three different HLA-B*07:02positive mumps patients. Interestingly, the first described MuV-specific CD4 ${ }^{+}$T-helper cell epitope, GTYRLIPNARANLTA $\left(\mathrm{NP}_{110}-124\right)$, which we recently discovered ${ }^{18}$, contains the newly identified HLA$B^{\star 07}$ :02-restricted $C D 8^{+} T$ cell epitope IPNARANL $\left(N P_{115-122}\right)$. This opens the possibility of using synthetic peptides, containing both epitope sequences, to stimulate $\mathrm{CD} 4^{+}$and $\mathrm{CD} 8^{+} \mathrm{T}$ cell responses simultaneously. In addition to the HLA-B*07:02-restricted epitopes, HLA-A*01:01-restricted T cell epitopes (i.e. YSDPNNHEVY $\left(L_{1336-1343}\right)$ and VTDSNLIY $\left.\left(L_{1983-1992}\right)\right)$ showed to induce a T cell response in HLA$A^{\star}$ 01:01 positive mumps patients. Four out of the five novel MuV epitopes of the genotype $G$ outbreak strain (MuVi/Utrecht.NLD/40.10) appeared to be conserved among the two Jeryl Lynn vaccine strains (JL2 and JL5), and therefore are useful for monitoring cellular immune responses after natural infection with the genotype $\mathrm{G}$ outbreak strain and after vaccination. Interestingly, one of the HLA-B*07:02-restricted epitopes, i.e. APIQGTNLL (V/P/I $27-35)$ showed to have sequence differences among various circulating mumps strains, including genotype $\mathrm{G}, \mathrm{H}$ and $\mathrm{C}$ strains, and also differ from the peptide sequences in the two Jeryl Lynn vaccine strains (JL2 and JL5). Future experiments are planned to explore the impact of this mismatch on the $T$ cell recognition of the various MuV strains, i.e. vaccine versus circulating MuV strains. Using peptide-HLA class I ( $\mathrm{p}: \mathrm{HLA}-\mathrm{I})$ dextramers, high frequencies of T cells against all five MuV epitopes were ex vivo detected in PBMCs of all three HLA-B*07:02- and three HLA-A*01:01-positive mumps patients. Functional characterization of these specific $T$ cells revealed a cytotoxic phenotype with expression of degranulation marker CD107a, and secretion of granzyme-A/B, granulysin, perforin, sFas(L). With a flow cytometry-based cytotoxicity assay, epitope-specific $T$ cells showed indeed to be able to efficiently lyse MuV peptide-pulsed target cells. Thus, we for the first time describe that MuV epitopespecific $C D 8^{+}$effector $T$ cells isolated from mumps patients have a cytotoxic phenotype which is a critical feature for mediating viral clearance following acute MuV infection. Although T cells against the novel MuV epitopes were also detectable in recently vaccinated persons, only very low frequencies of 
these specific $C D 8^{+} T$ cells were observed, which is in line with our previous findings ${ }^{9}$. Live-attenuated vaccines, such as MMR vaccines, are likely to be able to induce $C D 8^{+} T$ cells, but it might be that the vaccine-induced $C D 8^{+} T$ cell response is low due to attenuated replication of the vaccine virus. As a result, the mumps-specific CD4/CD8 $\mathrm{T}$ cell ratio may be lower after vaccination than after natural infection, as confirmed in our previous study ${ }^{9}$. Both $\mathrm{CD} 4^{+}$and $\mathrm{CD} 8^{+} \mathrm{T}$ cells are involved in protection against viral disease. Prevention of infection can be achieved by induction of $C D 4^{+} T$ cells providing help to $B$ cells to produce protective virus-neutralizing antibodies, whereas vaccine-induced $C D 8^{+} \mathrm{T}$ cells support disease attenuation and protection against complications after infection ${ }^{19}$.

In order to be able to accurately measure the low $\mathrm{CD} 8^{+} \mathrm{T}$ cell response after vaccination, we propose that not a single peptide but rather a pool of confirmed immunogenic peptides representing a broad HLA-I coverage should be used. Such a peptide pool would provide a useful tool to monitor and functionally characterize the $\mathrm{CD} 8^{+} \mathrm{T}$ cell response to $\mathrm{MuV}$ to better understand its role after natural infection and vaccination.

\section{Methods}

\section{Subjects and blood sample handling}

Peripheral blood samples of mumps patients were collected within the first month after disease as part of the clinical study VAC-263 (NL37852.094) ${ }^{20}$. In addition, peripheral blood samples were used that were collected from young adults (18-30 years) four weeks after they received a third dose of the MMR vaccine as part of our MMR3 clinical study ${ }^{6}$ or from our blood donor system ${ }^{21}$. Written informed consent was obtained for all participants. The clinical studies were approved by the medical ethical committee (METC Noord-Holland) and performed in accordance with the Declaration of Helsinki.

HLA class I typing ( $B * 07: 02$ and HLA-A*01:01) was performed by high resolution next-generation sequencing (University Medical Center Utrecht, the Netherlands) or by flow cytometry using anti-HLA class I A1 antibody (8.L.104, biotin-labeled) or anti-HLA class I B7 antibody (BB7.1, PE-labeled (both Abcam) utilizing LSRFortessaX20 (BD) and analyzed with FlowJo (V10/Treestar). Peripheral blood mononuclear cells (PBMCs) were isolated from the blood samples by Ficoll-Hypaque gradient centrifugation and cryopreserved. Epstein-Barr virus-transformed B-lymphoblastoid cell lines (BLCL) as antigen-presenting cells, were generated from PBMCs as described before ${ }^{21}$.

\section{Prediction of binding of MuV peptides to HLA-A*01:01 and $B * 07: 02$ molecules}

The 41 MuV peptides, that were previously eluted from HLA class I molecules of MuV-infected cells ${ }^{16}$, were tested for predicted binding to the (common) HLA-A*01:01 and $B \star 07: 02$ molecules using the NetMHCpan 4.0 server. A threshold for selection of best-binding peptides was set on rank $<2 \% 22$. 


\section{Generation of effector $\mathrm{CDB}^{+} \mathrm{T}$ cell lines}

In order to generate effector $C D 8^{+} T$ cell lines, PBMCs from three HLA-B*07:02 positive and three HLA$A * 01: 01$ positive mumps patients were thawed. Cells were stained with fluorochrome-conjugated antibodies anti-CD3-APC/R700, anti-CD4-BV711, anti-CD8-BV786, anti-CD45RO-PE/Cy7 (all from BD Biosciences), and CCR7-PerCP/Cy5.5 (BioLegend). A subset of the effector T cells (CD3 ${ }^{+}, \mathrm{CD}^{+}, \mathrm{CCR}^{-}$, $\mathrm{CD}^{4} \mathrm{RO}^{-}$) was sorted using FACSMelody sorter (BD) in order to increase MuV-specific T cell frequencies for peptide immunogenicity testing. Sorted T cells were further expanded in AIM-V medium (Gibco) supplemented with $2 \%$ human AB serum, with irradiated (3000 rad) allogeneic PBMCs and irradiated (5000 rad) allogeneic BLCL cells, $1 \mu \mathrm{g} / \mathrm{ml}$ phytohemagglutinin (Sigma), and $5 \mathrm{ng} / \mathrm{ml} \mathrm{IL}-2$. Every 3-4 days IL-2 (5 ng/ml) was added. Cells were frozen when they reached a total of $\geq 2 \times 10^{6}$.

\section{Immunogenicity testing for $\mathrm{CD8}^{+} \mathrm{T}$ cell epitope identification}

Thawed CD8 ${ }^{+}$T-effector cell lines were cultured for 6 hours in AIM-V containing $2 \%$ human AB serum in the presence of one of the selected best-binding peptides $(1 \mu \mathrm{M}$; synthesized by JPT Peptide Technologies Inc). During the last 4 hours, Brefeldin A and Monensin (BD) were added. Stimulated cells were stained with fluorochrome-conjugated antibodies, anti-CD3-BUV395, anti-CD4-BV711, anti-CD8BV786, and Fixable Viability Stain 780 (all BD Biosciences). For intracellular staining, cells were fixed, permeabilized and stained with anti-IFNY-APC (BD) and anti-TNF-FITC (eBioscience/ThermoFisher), using the Foxp3/transcription factor staining buffer set (eBioscience/ThermoFisher). Cells were acquired on an LSRFortessaX20 (BD) and analyzed using FlowJo (V10/Treestar).

Ex vivo detection of MuV epitope-specific CD8 ${ }^{+} \mathrm{T}$ cells from mumps patients and vaccinees

Five immunogenic peptides were selected that were able to induce a T cell response. Subsequently, fluorochrome-labeled peptide-HLA class I ( $\mathrm{p}: \mathrm{HLA}-\mathrm{I}-$ ) dextramers each loaded with one of these five peptides were synthesized: $B^{\star}$ 0702-APIQGTNLL (FITC-labeled), $B^{\star}$ 0702-KPRTSTPVTEF (PE-labeled), $B * 0702-I P N A R A N L$ (APC-labeled), $A * 0101-Y S D P N N H E V Y$ (PE-labeled), and $A * 0101-V T D S N L I Y$ (APClabeled) (all Immudex). In order to detect ex vivo MuV-specific CD8 ${ }^{+} \mathrm{T}$ cells, $4 \times 10^{6}$ PBMCs from three HLA-B*0702- and three HLA-A*0101-positive mumps patients and from two HLA-B7- and two HLA-A1positive young adults that recently received a third dose of the MMR vaccine, were stained with a combination of the different $\mathrm{p}$ :HLA-I-dextramers and incubated for 10 minutes at room temperature. Subsequently, cells were stained with anti-CD3, anti-CD4, anti-CD8, anti-CD45RO and anti-CCR7 for 15 minutes at $4{ }^{\circ} \mathrm{C}$. Cells were acquired on an LSRFortessaX20.

\section{Expansion of MuV epitope-specific CD8 ${ }^{+} \mathrm{T}$ cells from mumps patients}


Epitope-specific $\mathrm{CD}^{+} \mathrm{T}$ cell lines were generated by sorting dextramer-positive cells (FACSMelody sorter (BD)) from HLA-B*0702- and HLA-A*0101-positive mumps patients and subsequent further expanded as described above for effector $\mathrm{CD} 8+\mathrm{T}$ cell lines.

\section{Functional characterization of epitope-specific $\mathrm{CD}^{+} \mathrm{T}$ cells Cytokine secretion analysis}

Cytokine release of epitope-specific $T$ cell lines was measured after stimulation with one of the five selected peptides $(5 \mu \mathrm{M})$ or medium as negative control, and performed in triplicate. Cell-free culture supernatant was collected to determine quantitating levels of IL-2, IL-4, IL-6, IL-10, IL-17A, TNF, IFN- $\gamma$, granzyme A (GrA), granzyme B (GrB), perforin, soluble Fas ligand (sFasL), soluble Fas (sFas), and granulysin in a multiplex bead-based assay (LEGENDplex human CD8/NK panel; BioLegend) according to the manufacturers' instructions and using FACSCantoll (BD).

\section{Testing for expression of functional markers}

Epitope-specific cell lines were stained with anti-CD3, anti-CD4, anti-CD8, anti-CD45RO and anti-CCR7 for 15 minutes at $4{ }^{\circ} \mathrm{C}$. Anti-CD107a-BV421 (BioLegend) was added during culture. For intracellular staining, cells were fixed, permeabilized, and stained with anti-CD137-PE, anti-IFN- $\gamma$ and anti-TNF using the Foxp3/transcription factor staining buffer set. Cells were acquired on an LSRFortessaX20.

\section{Testing for cytotoxicity capacity}

A flow cytometry-based killing assay was used to determine the cytotoxic capacity of the various epitopespecific $T$ cell lines ${ }^{18}$. For this purpose, BLCL as antigen-presenting cells were labeled with CellTrace violet (Life Technologies) and pulsed with one of the five selected peptides $(5 \mu \mathrm{M})$ or medium as negative control. Subsequently, epitope-specific T cell lines and BLCL were co-cultured at different effector/target $(E: T)$ ratios in AIM-V supplemented with $2 \%$ human $A B$ serum. After 4 hours, cells were stained with Fixable Viability Stain 780 (BD) and fixated. Cells were acquired using a LSRFortessa X20. Measurements were made in triplicate and averaged.

\section{Declarations}

\section{Acknowledgements}

We are grateful to Jacqueline van Gaans for helping with peptide elution experiments.

\section{Author information}

\section{Affiliations}

Centre for Infectious Disease Control, National Institute for Public Health and the Environment, Bilthoven, the Netherlands 
Patricia Kaaijk, Maarten E. Emmelot, Cécile A.C.M. van Els, Jelle de Wit ${ }^{1}$

Intravacc (Institute for Translational Vaccinology), Bilthoven, The Netherlands

Hugo D. Meiring,

\section{Contributions}

P.K. conceived the project; P.K., J.W. and C.E. designed the study; M.E., H.D.M., P.K, and J.W. planned experiments; M.E. and H.D.M. performed experiments; M.E., P.K. and J.W. analyzed data; M.E. and P.K. prepared figures; P.K. wrote first draft of the manuscript and all authors contributed to the final version of the paper.

\section{Corresponding author}

Correspondence to Patricia Kaaijk

\section{Ethics Declaration}

\section{Competing interests}

The authors declare no competing interests.

\section{Financial support}

This work was supported by the Dutch Ministry of Health, Welfare and Sport.

\section{Data availability}

NetMHCpan-4.0 server ${ }^{22}$ was used to predict binding of the set of 41 mumps virus (MuV) peptides to the HLA-A*01:01 and $B \star 07: 02$ molecules. This server is available via https://services.healthtech.dtu.dk/service.php?NetMHC-4.0

\section{References}

1 Bankamp, B., Hickman, C., Icenogle, J. P. \& Rota, P. A. Successes and challenges for preventing measles, mumps and rubella by vaccination. Curr Opin Viro/34, 110-116, doi:10.1016/j.coviro.2019.01.002 (2019).

2 Rubin, S., Kennedy, R. \& Poland, G. Emerging Mumps Infection. Pediatr Infect Dis J35, 799-801, doi:10.1097/INF.0000000000001182 (2016).

3 Dayan, G. H. et al. Recent resurgence of mumps in the United States. N Eng/ J Med358, 1580-1589 (2008). 
4 Cohen, C. et al. Vaccine effectiveness estimates, 2004-2005 mumps outbreak, England. Emerg Infect Dis13, 12-17 (2007).

5 Kontio, M., Jokinen, S., Paunio, M., Peltola, H. \& Davidkin, I. Waning antibody levels and avidity: implications for MMR vaccine-induced protection. J Infect Dis206, 1542-1548, doi:10.1093/infdis/jis568 (2012).

6 Kaaijk, P. et al. A Third Dose of Measles-Mumps-Rubella Vaccine to Improve Immunity Against Mumps in Young Adults. J Infect Dis221, 902-909, doi:10.1093/infdis/jiz188 (2020).

7 Smits, G. et al. Seroprevalence of mumps in The Netherlands: dynamics over a decade with high vaccination coverage and recent outbreaks. PLoS One8, e58234, doi:10.1371/journal.pone.0058234 (2013).

8 Kaaijk, P. et al. Dynamics of the Antibody Response After a Third Dose of Measles-Mumps-Rubella Vaccine Indicate a Slower Decline Compared With a Second Dose. Open Forum Infect Dis7, ofaa505, doi:10.1093/ofid/ofaa505 (2020).

9 de Wit, J. et al. Mumps infection but not childhood vaccination induces persistent polyfunctional CD8(+) T cell memory. The Journal of allergy and clinical immunology141, 1908-1911 e1912, doi:10.1016/j.jaci.2017.11.047 (2018).

10 Jokinen, S., Osterlund, P., Julkunen, I. \& Davidkin, I. Cellular immunity to mumps virus in young adults 21 years after measles-mumps-rubella vaccination. J Infect Dis196, 861-867, doi:JID38157 [pii]

$10.1086 / 521029(2007)$.

11 Hanna-Wakim, R., Yasukawa, L. L., Sung, P., Arvin, A. M. \& Gans, H. A. Immune responses to mumps vaccine in adults who were vaccinated in childhood. $J$ Infect Dis197, 1669-1675, doi:10.1086/588195 (2008).

12 Vandermeulen, C. et al. Evaluation of cellular immunity to mumps in vaccinated individuals with or without circulating antibodies up to 16 years after their last vaccination. J Infect Dis199, 1457-1460, doi:10.1086/598482 (2009).

13 Rasheed, M. A. U. et al. Decreased humoral immunity to mumps in young adults immunized with MMR vaccine in childhood. Proc Natl Acad Sci U S A116, 19071-19076, doi:10.1073/pnas.1905570116 (2019).

14 Connell, A. R., Connell, J., Leahy, T. R. \& Hassan, J. Mumps Outbreaks in Vaccinated Populations-ls It Time to Re-assess the Clinical Efficacy of Vaccines? Front Immuno/11, 2089, doi:10.3389/fimmu.2020.02089 (2020).

15 Ramanathan, R., Voigt, E. A., Kennedy, R. B. \& Poland, G. A. Knowledge gaps persist and hinder progress in eliminating mumps. Vaccine36, 3721-3726, doi:10.1016/j.vaccine.2018.05.067 (2018). 
16 de Wit, J. et al. Identification of Naturally Processed Mumps Virus Epitopes by Mass Spectrometry: Confirmation of Multiple CD8+ T cell Responses in Mumps Patients. J Infect Dis221, 474-482, doi:10.1093/infdis/jiz480 (2020).

17 Gonzalez-Galarza, F. F. et al. Allele frequency net database (AFND) 2020 update: gold-standard data classification, open access genotype data and new query tools. Nucleic Acids Res48, D783-D788, doi:10.1093/nar/gkz1029 (2020).

18 de Wit, J. et al. The Human CD4(+) T Cell Response against Mumps Virus Targets a Broadly Recognized Nucleoprotein Epitope. J Viro/93, doi:10.1128/JVI.01883-18 (2019).

19 Siegrist, C. A. Vaccine Immunology (Chapter 2). In: Plotkin SA, Orenstein WA, Vaccines. 7th edn, (Elsevier Inc. Philadelphia, 2018).

20 Kaaijk, P. et al. Dynamics of the serologic response in vaccinated and unvaccinated mumps cases during an epidemic. Hum Vaccin Immunother11, 1754-1761, doi:10.1080/21645515.2015.1040967 (2015).

21 Han, W. G. et al. Development of an IFNgamma ELISPOT for the analysis of the human T cell response against mumps virus. J Immunol Methods431, 52-59, doi:10.1016/j.jim.2016.02.010 (2016).

22 Jurtz, V. et al. NetMHCpan-4.0: Improved Peptide-MHC Class I Interaction Predictions Integrating Eluted Ligand and Peptide Binding Affinity Data. J Immuno/199, 3360-3368, doi:10.4049/jimmunol.1700893 (2017).

\section{Figures}




\section{HLA-B*07:02}

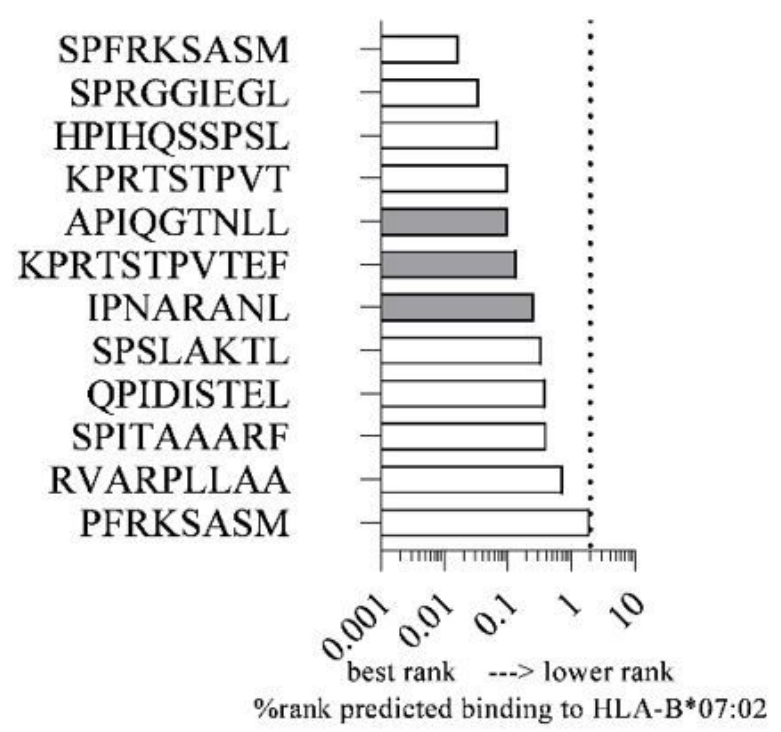

\section{HLA-A*01:01}

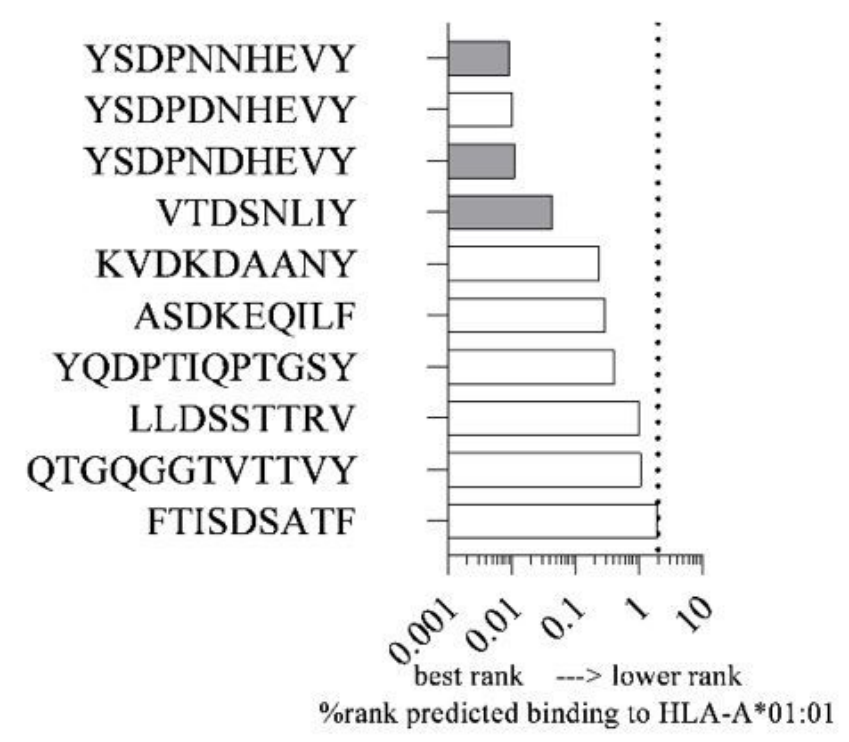

tested positive in $\mathrm{T}$ cell assay

\section{Figure 1}

Predicted binding affinity of the candidate epitopes to HLA and capability to induce cytokine production by $T$ cells is presented. Mumps virus (MuV) candidate epitopes were selected based on their predicted binding (threshold rank $<2 \%$ ) to HLA-B*07:02 molecule (left panel; $n=12$ peptides) and HLA-A*01:01 molecule (right panel; $n=10$ peptides). Selected peptides were used to stimulate peripheral blood mononuclear cells (PBMC) of respectively three HLA-B*0702 positive and three HLA-A*01:01 positive mumps patients. $C D 8+T$ effector cell lines from these patients were stimulated with the selected peptides and production of interferon (IFN)- $\gamma$ and tumor necrosis factor (TNF) by CD8+ T cells was measured by flow cytometry. Responding T cells were identified as a 2-fold increase of IFN $\gamma+/ T N F+$ cells within the $\mathrm{CD} 3+/ \mathrm{CD} 4-/ \mathrm{CD} 8+$ live gate. Peptides that were tested positive, induced a response in all three HLA-B*07:02 and HLA-A*01:01 positive mumps patients. Filled bars (gray) indicate the peptides that tested positive. 
APIQGTNLL
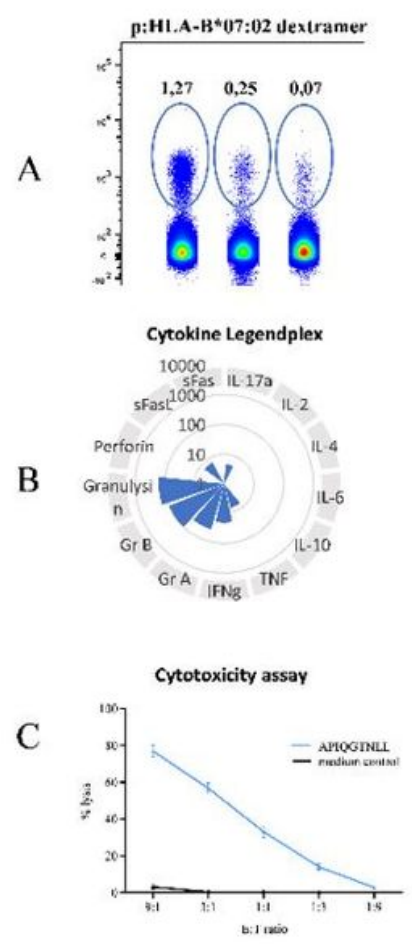

KPRTSTPVTEF
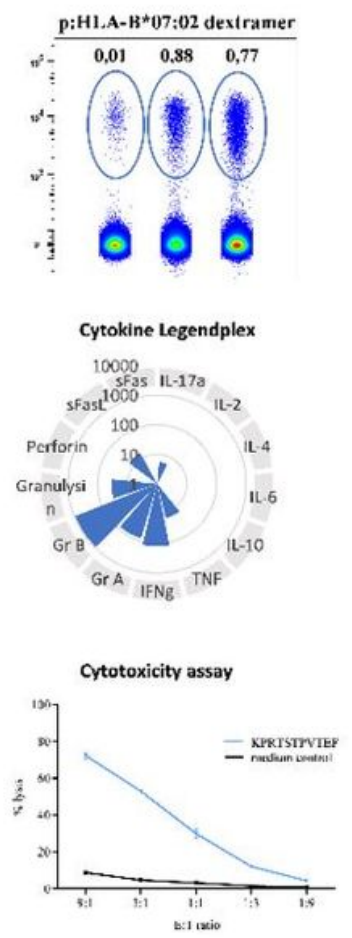

IPNARANL
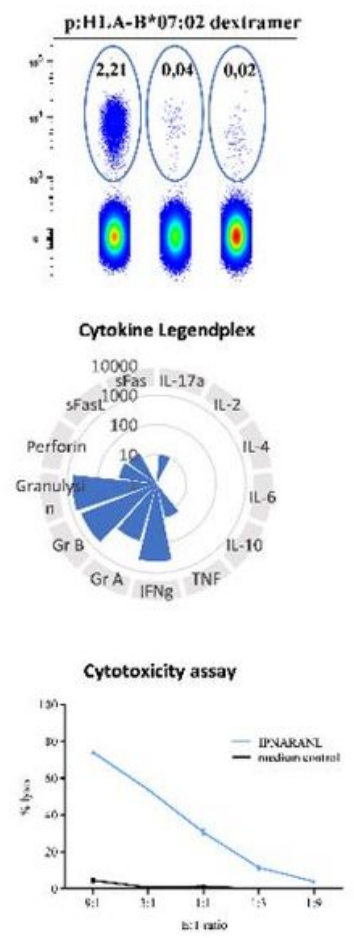

YSDPNNHEVY

VTDSNLIY
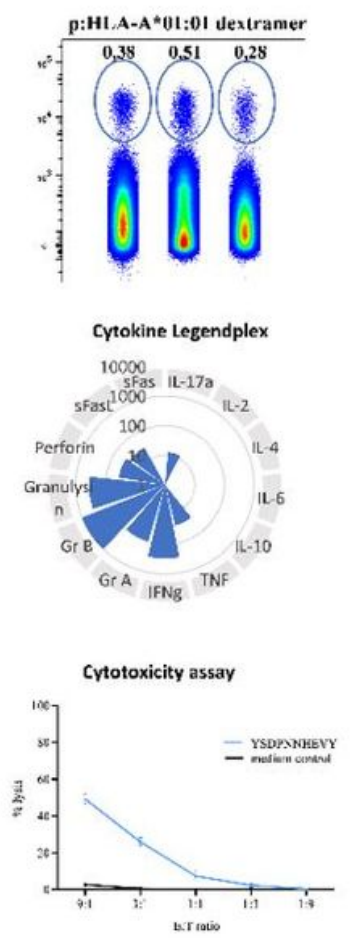
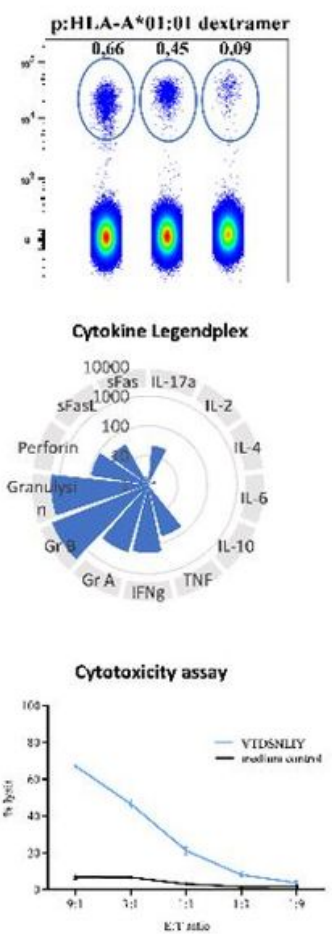

Figure 2

Detection and functional characterization of epitope-specific T cells from mumps patients. (A) Flow cytometric ex vivo detection of epitope-specific T cells using p:HLA-I dextramers in T cellPBMC samples of three HLA-B*07:02 patients (\#03, \#08 and \#20) and three HLA-A*01:01-positive mumps patients (\#04, \#14 and \#25). (B) Cytokine analysis of epitope-specific T cell lines derived from HLA-B*07:02- or HLA$A \star 01: 01$-positive mumps patients measured with a multiplex bead-based assay quantitating levels of interleukin-2 (IL-2), IL-4, IL-6, IL-10, tumor necrosis factor (TNF), interferon gamma (IFN-ץ), granzyme A (GrA), granzyme B (GrB), perforin, soluble Fas ligand (sFasL), soluble Fas (sFas), and granulysin. Concentrations of measured cytokines are all expressed as means of triplicates in $\mathrm{pg} / \mathrm{mL}$. (C) Cytotoxic activity of epitope-specific $T$ cell lines isolated from HLA-B*07:02- or HLA-A*01:01-positive mumps patients measured with a flow cytometry-based killing assay. Specific lysis, presented as mean of triplicates \pm SD, was calculated as follows: \% specific lysis $=[(\%$ dead target cells $-\%$ spontaneous dead target cells)/ (100 - \% spontaneous dead target cells)] $\times 100 \%$ 
APIQGTNLL

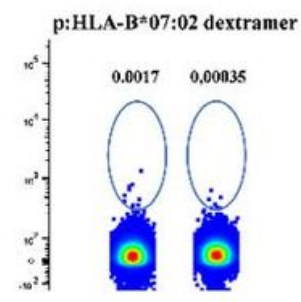

KPIRTSTPVTEF

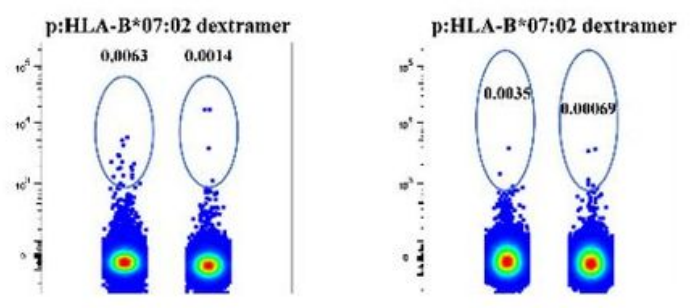

YSDPNNHEVY

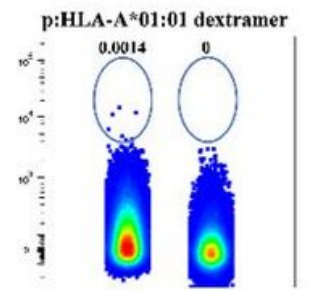

VTDSNLIY

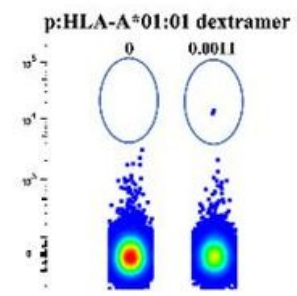

Figure 3

Detection of epitope-specific T cells from recently vaccinated young adults. (A) Flow cytometric ex vivo detection of epitope-specific T cells using p:HLA-I dextramers from PBMC samples of two HLA-B7positive (\#IIV-291-034 and VAC-076) and two HLA-A1-positive vaccinees (\#IIV-291-002 and \#025).

Page 13/13 